NOTE

\title{
Species identification of two sympatric hakes by allozymic markers*
}

\author{
M. I. ROLDÁN and C. PLA \\ Laboratori d’Ictiologia Genètica, Universitat de Girona, Campus Montilivi, E-17071 Girona, Spain. FAX: 34-972-418277. \\ E-mail: dbmrb@fc.udg.es
}

\begin{abstract}
SUMMARY: Samples were collected from hake species Merluccius australis and M. hubbsi in the south west Atlantic Ocean. Enzyme electrophoretic analysis of the eye, liver and muscle revealed 5 out of 33 genetic loci with species-specific allelic frequencies. These five loci provide a set of genetic markers for individual classification.
\end{abstract}

Key words: identification, allozymes, hake, Merluccius australis, M. hubbsi, southwest Atlantic Ocean.

\section{INTRODUCTION}

The morphological similarities among species and the uncertainty of phylogenetic relationships suggest the application of molecular techniques. These methods have provided critical insights towards resolving similar problems in teleost taxa. Allozyme electrophoresis has been a widely applied molecular method for comparing levels of genetic divergence between populations and between taxa (e.g. Grant et al., 1999). The findings have direct implications on species management and taxa biogeography. Allozyme differences are also useful for inexpensive and uncomplicated species identification using isolated tissues (e.g. fish fillets), or intact individuals of morphologically indistinct taxa (Utter et al., 1974; Shaklee et al., 1982). These applications are the topic of this communication. Two southwestern Atlantic hakes, M. australis (Southern hake) and M. hubbsi (Argentinean hake), have over-

\footnotetext{
*Received April 18, 2000. Accepted July 27, 2000.
}

lapping distributions in the austral zone of the Argentinean Sea, with intense commercial harvesting throughout the ranges of both species (Fig. 1). Immature fish of these species are difficult to distinguish by simple observation, and a portion of catches reported as Argentinean hake are believed to be Southern hake (FAO, 1997). Data presented in this work provide a tool for resolving the identification of catches reported from the south-west Atlantic fishery.

\section{MATERIALS AND METHODS}

We examined morphologically distinct adults of both species (86 Argentinean hake and 24 Southern hake) captured together in the Argentine Sea (Fig. 1) by Instituto Nacional de Investigación y Desarrollo Pesquero, Mar del Plata, Argentina. They were immediately frozen with dry ice and stored at $-80^{\circ} \mathrm{C}$ prior to electrophoretic analysis. Tissue extraction, electrophoresis and procedures for visualising pro- 


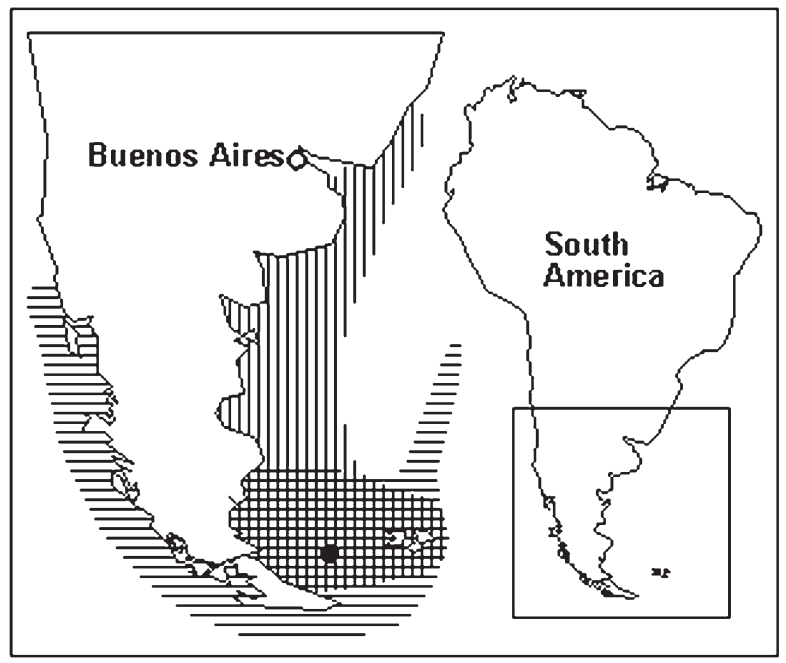

FIG. 1. - Distribution of Merluccius australis (horizontal lines) and M. hubbsi (vertical lines) from Cohen et al., 1990. Solid circle: sampling site.

teins followed the methods outlined in Roldán et al. (1998). Extracts from eye, liver and skeletal muscle were electrophoretically screened for resolution and activity with buffer systems described in GarcíaMarín et al. (1991) (Table 1). Genetic nomenclature follows Shaklee et al. (1990). Alleles were designated by their mobilities relative to the most common allele in $M$. hubbsi, which was designated $* 100$ for each locus. All data analysis was computed by BIOSYS (Swofford and Selander, 1981).

\section{RESULTS AND DISCUSSION}

We interpreted the banding patterns of 20 enzyme systems to be a reflection of 33 genetic loci (Table 1). Fifteen out of 33 were monomorphic, based on the identical phenotypes for all individuals tested in both species. Variation was detected for the remaining 18 loci between species (Table 2). The genetic distance (Nei, 1972) 0.157 indicated a close between-species relationship, compared to a distance of 0.583 (31 loci; Grant et al., 1988) between two sympatric South African hakes (M. capensis and $M$. paradoxus). The expected heterozygosity (Nei, 1978) for both species shows the highest values among the species' genera (M. australis $=0.099$; M. hubbsi=0.089) (Roldán et al., 1999).

Many of the loci are useful markers for identifying specific catches throughout allelic frequencies

TABLE 1. - Enzyme systems, loci abbreviations and tissues with strongest expression. M, skeletal muscle; L, liver; E, eye.

\begin{tabular}{|c|c|c|c|c|}
\hline Enzyme & E C no. & Loci & Tissue & Polymorphic \\
\hline Adenylate kinase & 2.7.4.3 & $A K^{*}$ & M & No \\
\hline Creatine kinase & 2.7 .3 .2 & $C K^{*}$ & M & Yes \\
\hline \multirow[t]{4}{*}{ Esterase } & 3.1.1.- & $E S T-1 *$ & $\mathrm{M}, \mathrm{E}$ & No \\
\hline & & $E S T-2 *$ & $\mathrm{M}, \mathrm{E}$ & Yes \\
\hline & & EST-3* & $\mathrm{M}, \mathrm{E}$ & No \\
\hline & & EST-4* & $\mathrm{M}, \mathrm{E}$ & Yes \\
\hline Glyceraldehyde-3-phosphate & 1.2.1.12 & GAPDH-1* & M & No \\
\hline \multirow[t]{2}{*}{ dehydrogenase } & & $G A P D H-2 *$ & $\mathrm{E}$ & No \\
\hline & & GAPDH-3* & $\mathrm{E}$ & No \\
\hline Glycerate dehydrogenase & 1.1.1.29 & GLYDH-1* & $\mathrm{L}$ & Yes \\
\hline Glycerol-3-phosphate dehydrogenase & 1.1.1.8 & $G 3 P D H^{*}$ & M & Yes \\
\hline \multirow{2}{*}{ Glucose-6-phosphate isomerase } & 5.3.1.9 & $G P I-1 *$ & M & Yes \\
\hline & & GPI-2* & $\mathrm{E}$ & Yes \\
\hline Glutathione reductase & 1.6.4.2 & $G R-2 *$ & $\mathrm{~L}$ & Yes \\
\hline \multirow[t]{2}{*}{ Isocitrate dehydrogenase } & 1.1.1.42 & $I D H P-1 *$ & M & Yes \\
\hline & & $I D H P-2 *$ & $\mathrm{~L}, \mathrm{E}$ & Yes \\
\hline \multirow[t]{3}{*}{ L-Lactate dehydrogenase } & 1.1.1.27 & $L D H-A^{*}$ & M & No \\
\hline & & $L D H-B^{*}$ & M & No \\
\hline & & $L D H-C^{*}$ & $\mathrm{~L}$ & No \\
\hline Lactoylglutathione lyase & 4.4.1.5 & $L G L^{*}$ & M & No \\
\hline \multirow[t]{2}{*}{ Malate dehydrogenase } & 1.1.1.37 & $M D H-2 *$ & M & No \\
\hline & & $M D H-3 *$ & M, L & No \\
\hline \multirow[t]{3}{*}{ Malic enzyme (NADP+) } & 1.1 .1 .40 & $M E P-1 *$ & M & Yes \\
\hline & & $M E P-2 *$ & M & No \\
\hline & & $M E P-3 *$ & $\mathrm{~L}, \mathrm{E}$ & Yes \\
\hline Peptidase-A (Glycyl-Leucine) & 3.4.-.- & $P E P-A^{*}$ & $\mathrm{M}, \mathrm{E}$ & Yes \\
\hline \multirow{2}{*}{ Peptidase-B (Leucyl-Glycyl-Glycine) } & 3.4.-.- & $P E P-B-1 *$ & $\mathrm{M}, \mathrm{E}$ & Yes \\
\hline & & $P E P-B-2 *$ & $\mathrm{M}, \mathrm{E}$ & No \\
\hline Peptidase-S (Leucyl-Tyrosine) & 3.4.-.- & $P E P-S-1 *$ & $\mathrm{M}, \mathrm{E}$ & Yes \\
\hline Phosphogluconate dehydrogenase & 1.1.1.44 & $P G D H^{*}$ & M & Yes \\
\hline Phosphoglucomutase & 5.4.2.2 & $P G M^{*}$ & M & Yes \\
\hline Pyruvate kinase & 2.7.1.40 & $P K-3 *$ & L & No \\
\hline Superoxide dismutase & 1.15.1.1 & $S O D^{*}$ & $\mathrm{~L}, \mathrm{E}, \mathrm{M}$ & Yes \\
\hline
\end{tabular}


TABLE 2. - Allelic frequencies of 18 polymorphic loci of $M$. australis and M. hubbsi from the Argentinian Sea. (N).

\begin{tabular}{|c|c|c|c|}
\hline Locus & allele & $\begin{array}{c}\text { M. australis } \\
\text { (24) }\end{array}$ & $\begin{array}{c}\text { M. hubbsi } \\
\text { (86) }\end{array}$ \\
\hline$C K^{*}$ & $\begin{array}{l}* 100 \\
* 115\end{array}$ & $\begin{array}{l}0.729 \\
0.271\end{array}$ & 1.000 \\
\hline \multirow[t]{3}{*}{ EST-2* } & $* 90$ & 0.979 & 0.250 \\
\hline & $* 100$ & 0.021 & 0.430 \\
\hline & $* 110$ & & 0.320 \\
\hline \multirow[t]{4}{*}{ EST-4* } & $* 90$ & & 0.198 \\
\hline & $* 95$ & 0.375 & \\
\hline & $* 100$ & 0.625 & 0.750 \\
\hline & $* 110$ & & 0.052 \\
\hline \multirow[t]{2}{*}{ GLYDH-1* } & $* 100$ & 1.000 & 0.994 \\
\hline & $* 115$ & & 0.006 \\
\hline \multirow[t]{2}{*}{$G 3 P D H^{*}$} & $* 100$ & & 0.959 \\
\hline & $* 110$ & 1.000 & 0.041 \\
\hline \multirow[t]{3}{*}{ GPI-1* } & $*-200$ & 0.021 & 0.012 \\
\hline & $* 100$ & 0.958 & 0.977 \\
\hline & $* 300$ & 0.021 & 0.012 \\
\hline \multirow[t]{3}{*}{ GPI-2* } & $* 85$ & 0.063 & 0.006 \\
\hline & $* 100$ & 0.917 & 0.977 \\
\hline & $* 125$ & 0.021 & 0.017 \\
\hline \multirow[t]{2}{*}{$G R-2 *$} & $* 83$ & & 0.006 \\
\hline & $* 100$ & 1.000 & 0.994 \\
\hline \multirow[t]{3}{*}{$I D H P-1 *$} & $* 65$ & 0.021 & \\
\hline & $* 75$ & 0.083 & 0.093 \\
\hline & $* 100$ & 0.896 & 0.907 \\
\hline \multirow[t]{3}{*}{$I D H P-2 *$} & $* 100$ & 0.021 & 0.884 \\
\hline & $* 115$ & 0.875 & 0.116 \\
\hline & $* 135$ & 0.104 & \\
\hline \multirow[t]{2}{*}{$M E P-1 *$} & $* 95$ & 0.083 & \\
\hline & $* 100$ & 0.917 & 1.000 \\
\hline \multirow[t]{2}{*}{$M E P-3 *$} & $* 80$ & & 0.006 \\
\hline & $* 100$ & 1.000 & 0.994 \\
\hline \multirow[t]{3}{*}{$P E P-A^{*}$} & $* 85$ & & 0.017 \\
\hline & $* 100$ & 0.854 & 0.983 \\
\hline & $* 105$ & 0.146 & \\
\hline \multirow[t]{2}{*}{$P E P-B-1 *$} & $* 80$ & 0.979 & 0.006 \\
\hline & $* 100$ & 0.021 & 0.994 \\
\hline \multirow[t]{3}{*}{$P E P-S-1 *$} & $* 95$ & & 0.140 \\
\hline & $* 100$ & 0.896 & 0.849 \\
\hline & $* 110$ & 0.104 & 0.012 \\
\hline \multirow[t]{12}{*}{$P G D H^{*}$} & $* 20$ & 0.813 & \\
\hline & $* 28$ & 0.167 & \\
\hline & $* 32$ & 0.021 & 0.006 \\
\hline & $* 55$ & & 0.076 \\
\hline & $* 75$ & & 0.314 \\
\hline & $* 90$ & & 0.029 \\
\hline & $* 100$ & & 0.267 \\
\hline & $* 110$ & & 0.017 \\
\hline & $* 120$ & & 0.076 \\
\hline & $* 130$ & & 0.134 \\
\hline & $* 145$ & & 0.035 \\
\hline & $* 155$ & & 0.047 \\
\hline \multirow[t]{4}{*}{$P G M^{*}$} & $* 70$ & & 0.006 \\
\hline & $* 90$ & 0.063 & 0.029 \\
\hline & $* 100$ & 0.833 & 0.936 \\
\hline & $* 110$ & 0.104 & 0.029 \\
\hline \multirow[t]{4}{*}{$S O D^{*}$} & $* 100$ & & 0.959 \\
\hline & $* 160$ & 0.271 & 0.041 \\
\hline & $* 230$ & 0.708 & \\
\hline & $* 315$ & 0.021 & \\
\hline
\end{tabular}

(see Table 2). Distinct allelic frequencies at five loci $\left(G 3 P D H^{*}, I D H P-2^{*}, P E P-B-I^{*}, P G D H^{*}\right.$ and $\left.S O D^{*}\right)$ provide a set of genetic markers for individual classification. However, joint analysis can be made from a single gel without additional effort. We suggest combined genotyping at $G 3 P D H^{*}$ and $P G D H^{*}$ muscular loci on $\mathrm{AC}(\mathrm{pH}=7)$ buffer as a fast identification method. This two locus screening makes it possible to distinguish occasional intraspecies polymorphism (heterozygosity at one or the other locus) from interspecies hybridisation (heterozygosity at both loci). This method will distinguish frozen and fresh processed fish, such as uncooked minced and fillets or ambiguous whole fishes of $M$. australis and $M$. hubbsi with almost complete confidence.

This current work also presents the results of an extensive search for electrophoretically detectable enzymatic loci in muscle, liver and eye of $M$. australis and $M$. hubbsi, which would serve as the basis for a deeper analysis that would provide greater knowledge of the population structure of both species. At this moment, despite their economic importance, few allozymic data useful for population differentiation are available on these two species. Roldán (1991) working with muscle on $M$. hubbsi reported four polymorphic loci and Smith et al. (1979) reported two polymorphic loci on M. australis from New Zealand.

\section{ACKNOWLEDGEMENTS}

We thank B. Jerez and A. Malaspina (INIDEP, Argentina) for helping to collect samples and J.L. García-Marín and F. Utter for helpful comments on the manuscript.

\section{REFERENCES}

Cohen, D.M., T. Inada, T. Iwamoto and N. Scialabba. - 1990. FAO species catalogue. Gadiform fishes of the world (Order Gadiformes). An annotated and illustrated catalogue of cods, hakes, grenadiers and other gadiform fishes known to date. FAO Fisheries Synopsis, no. 125, vol. 10, Rome, FAO, 442 pp.

FAO. - 1997. Review of the state of world fishery resources: marine fisheries. 6. Southwest Atlantic. FAO Fisheries circular No. 920 FIRM/C920. Rome, FAO.

García-Marín, J.L., P.E. Jorde, N. Ryman, F.M. Utter and C. Pla. 1991. Management implications of genetic differentiation between native and hatchery populations of brown trout (Salmo trutta) in Spain. Aquaculture, 95: 235-249.

Grant, W.S., I.I. Becker and R.W. Leslie. - 1988. Evolutionary divergence between sympatric species of southern African Hakes, Merluccius capensis and M. paradoxus. I. Electrophoretic analysis of proteins. Heredity, 61: 13-20.

Grant, W.S., J.L. García-Marín and F.M. Utter. - 1999. Defining population boundaries for fishery management. In: S. Mustafa (ed.) Genetics in sustainable fisheries management. pp. 27-72. Fishing New Books, Blackwell Science Ltd., Oxford.

Nei, M. - 1972. Genetic distance between populations. Am. Nat., 106: 283-292.

Nei, M. - 1978. Estimation of average heterozygosity and genetic distance from a small number of individuals. Genetics, 41: 225-233. 
Roldán, M.I. - 1991. Enzymatic polymorphisms in the Argentinian hake, Merluccius hubbsi Marini, of the Argentinian continental shelf. J. Fish Biol., 39: 53-59.

Roldán, M.I., J.L. García-Marín, F.M. Utter and C. Pla. - 1998. Population genetic structure of European hake, Merluccius merluccius. Heredity, 81: 327-334.

Roldán, M.I., J.L. García-Marín, F.M. Utter and C. Pla. - 1999. Genetic relationships among Merluccius species. Heredity, 83: 79-86.

Shaklee, J.B., C.S. Tamaru and R.S. Waples. - 1982. Speciation and evolution of marine fishes studied by the electrophoretic analysis of proteins. Pacific Science, 36: 141-157.

Shaklee, J.B., F.W. Allendorf, D.C. Morizot and G.S. Whitt. 1990. Gene nomenclature for protein-coding loci in fish. Trans. Amer. Fish. Soc., 119: 2-15.
Smith, P.J., G.J. Patchell and P.G. Benson. - 1979. Glucosephosphate isomerase and isocitrate dehydrogenase polymorphisms in the hake, Merluccius australis. N.Z. J. Mar. Fresh. Res., 13: 545-547.

Swofford, D.L. and R.B. Selander. - 1981. BIOSYS-1: a FORTRAN program for the comprehensive analysis of electrophoretic data in population genetics and systematics. $J$. Heredity, 72: 281-283.

Utter, F.M., H.O. Hodgins and F.W. Allendorf. - 1974. Biochemical genetic studies of fishes: potentialities and limitations. In: D.C. Malins and J.R. Sargent. (eds.) Biochemical and Biophysical Perspectives in Marine Biology. Volume I. pp. 213-237. Academic Press. London.

Scient. ed.: J. Lleonart 